\title{
Research on Automatic Section Precision Forming of Boom-type Roadheader
}

Ning Shurong, Su Hang ${ }^{a}$, Gao Jun ${ }^{b}$

School of Mechanical,Electrical \& Information Engineering, Shandong University,Weihai, China e-mail: ningshurong@163.com, ae-mail: suhang741@163.com ${ }^{\mathrm{b}} \mathrm{e}-\mathrm{mail}$ : shdgj@sdu.edu.cn

Keywords: boom-type roadheader; precision forming; approximate iterative method;

\begin{abstract}
The decision of the inflection point is the key to ensure automatic section cutting precision using a boom-type road header. In this paper, cutting precision model was set up for the first time to trapezoidal section and a method determining inflection point is given. Considered the cutting head' projection at section is elliptical with the cantilever's joint variable. The relationship between ellipse curve and cutting section boundary was investigated, and thereby sets up the cutting precision model. Approximate iterative method is used to finding the optimal inflection point, of which takes the cutting precision as the objective function. Compared with the traditional process path planning method, the forming accuracy can be improved greatly and controlled within $1 \mathrm{~mm}$. Through MATLAB simulation, it is proved that this method is correct and valid.
\end{abstract}

\section{Introduction}

Section automatic forming is known as an important research direction in the development of the automation of boom type roadheader[1]-[3]. Precise forming can obtain regular section shape and size, thereby reducing the useless amount of excavation and filling [4]. Completed the cutting operation along the process path, the envelope generated by the cutting head is profile curve of section. The forming accuracy of section is determined by the position of the inflection point on the process path; however, in most literatures[5]-[8], the inflection point is determined by simply using the method that boundary position minus the cutting head radius, which tends to produce less cutting or extra cutting. Through the establishment of pick coordinate, the literature[9] gives the formula of determining inflection point in the rectangular section, which can accurately calculate the projection coordinate on inflection point in the section, however, this method is not suitable for special section, such as trapezoidal. This paper proposed a method of determining the inflection point position in trapezoid section. First, the position relationship is established between the curve equation and the boundary of section based on the projection curve of cutting head, the approximate value of the inflection point is given, and then distance between the maximum cutting point formed by the pick and section boundary is taken as the objective function, finally, the optimal inflection point is found to ensure cutting precision by 'approximate iterative method'.

\section{The Establishment of Mathematical Model and the Operation Process Analysis of Boom-Type Roadheader}

A. The establishment of mathematical model of boom-type roadheader

Boom-type roadheader is mainly composed of a cutting head, a telescopic part, cantilever, frame, rotary table, a drive cylinder, hydraulic and electrical system etc. The boom-type roadheader's working device coordinate system and connecting rod parameters as shown in Fig.1. 


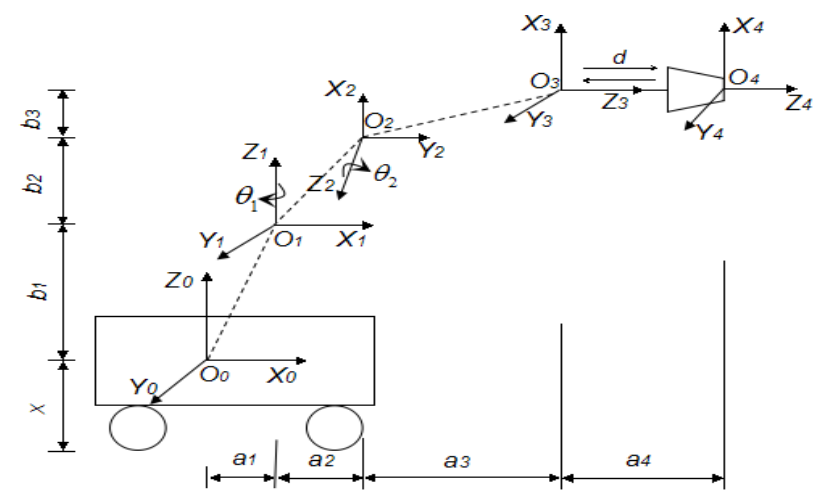

Figure 1. Roboticized roadheader model and connecting rod parameters

After solving for boom-type roadheader's working device kinematics, homogeneous transformation matrix of the end cutting head coordinates relative to the bodywork's coordinate system can be obtained:

$$
\begin{aligned}
{ }_{4}^{0} T & =\left[\begin{array}{cccc}
{ }_{0}^{0} T{ }_{2}^{1} T{ }_{3}^{2} T & { }_{4}^{3} T \\
c \theta_{1} c \theta_{2} & s \theta_{1} & -c \theta_{1} s \theta_{2} & \mathrm{~b}_{3} c \theta_{1} c \theta_{2}-\left(a_{3}+a_{4}+d\right) c \theta_{1} s \theta_{2}+a_{2} c \theta_{1}+a_{1} \\
s \theta_{1} c \theta_{2} & -c \theta_{1} & -s \theta_{1} s \theta_{2} & \mathrm{~b}_{3} s \theta_{1} c \theta_{2}-\left(a_{3}+a_{4}+d\right) s \theta_{1} s \theta_{2}+a_{2} s \theta_{1} \\
-s \theta_{2} & 0 & -c \theta_{2} & -\mathrm{b}_{3} s \theta_{2}-\left(a_{3}+a_{4}+d\right) c \theta_{2}+b_{2} \\
0 & 0 & 0 & 1
\end{array}\right]
\end{aligned}
$$

Where, $\theta_{1}, \theta_{2}$ and $d$ are the joint variables, a1, a2, a3, a4, b1, b2 and b3 are the connecting rod constants.

Coordinate vector of the cutting head's center point ${ }_{4}$ in the coordinate system $O_{4}-X_{4} Y_{4} Z_{4}$ is

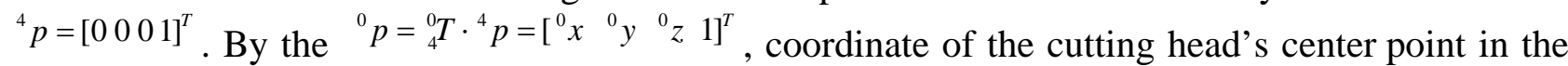
bodywork's coordinate system is:

$$
\left\{\begin{array}{l}
{ }^{0} x=b_{3} c \theta_{1} c \theta_{2}-\left(a_{3}+a_{4}+d\right) c \theta_{1} s \theta_{2}+a_{2} c \theta_{1}+a_{1} \\
{ }^{0} y=b_{3} s \theta_{1} c \theta_{2}-\left(a_{3}+a_{4}+d\right) s \theta_{1} s \theta_{2}+a_{2} s \theta_{1} \\
{ }^{0} z=-b_{3} s \theta_{2}-\left(a_{3}+a_{4}+d\right) c \theta_{2}+b_{2}
\end{array}\right.
$$

\section{B. The operation process analysis of boom-type roadheader}

The boom-type roadheader's cutting operation generally use the cutting process path as shown in fig.2 (with rectangular section as an example): Forming control of the boom-type roadheader based on path planning and trajectory planning. When the section is given in the forms and the specific size, according to the step length, first we can determine the coordinate sequence $\left\{\left(x_{0}, y_{0}\right),\left(x_{1}, y_{1}\right), \ldots . . .\left(x_{j}, y_{j}\right)\right\}$ of the inflection point in section coordinate planes, then according to the sequence of the path planning, straight line trajectory planning for two adjacent elements in turn. Thus we can get a S-shaped process path that traverses the entire presection.

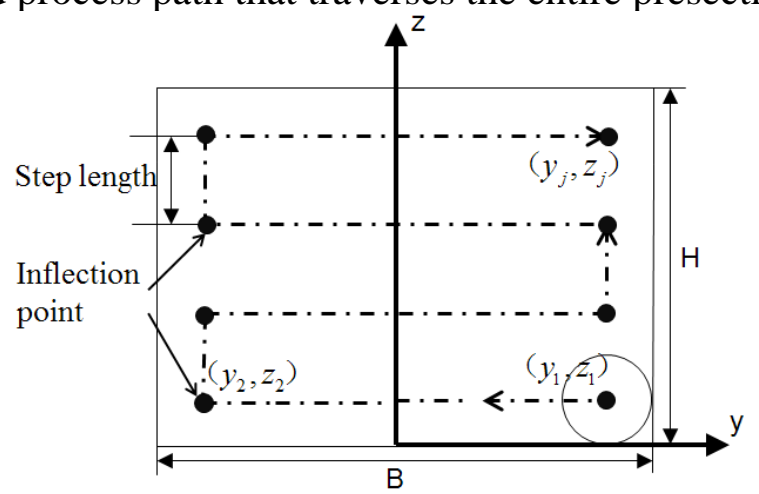

Figure 2. Enactment of cutting path 


\section{Precision Forming Theory of Sectional Contour}

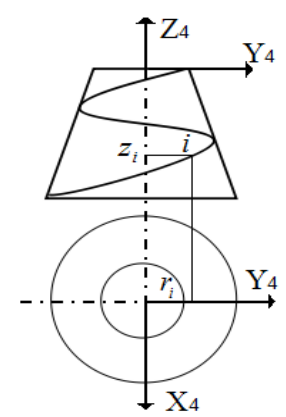

Figure 3. Pick coordinates system

After the section of the form given, section real boundary is formed when the projection curve of pick and the boundary of the predetermined section tangent. The position of the inflection point should be based on this principle to determine. Pick coordinate system is shown in Fig. 3, and set coordinate vectors of the pick i which formed the section boundary in the coordinate system $\mathrm{O}_{4}-X_{4} Y_{4} Z_{4}$ is:

${ }^{4} p=\left[\begin{array}{llll}{ }^{4} x_{i} & { }^{4} y_{i} & { }^{4} z_{i} & 1\end{array}\right]$

So, coordinate of the pick $\boldsymbol{i}$ relative to bodywork's coordinate system $O_{0}-X_{0} Y_{0} Z_{0}$ is:

${ }^{0} p={ }_{4}^{0} T \cdot{ }^{4} p=\left[\begin{array}{llll}{ }^{0} x_{i} & { }^{0} y_{i} & { }^{0} z_{i} & 1\end{array}\right]^{T}$

When roadheader do the cutting work, cantilever swing speed is much slower than cutting head rotating speed, thus cutting track of pick at each time point can be expressed as a circular trajectory around the cutting head axis. In the $\mathrm{O}_{4}-X_{4} Y_{4} Z_{4}$, parameter equation of the circular trajectory can be expressed as:

$$
\left\{\begin{array}{l}
{ }^{4} x_{i}=r_{i} s \theta \\
{ }^{4} y_{i}=r_{i} c \theta \\
{ }^{4} z_{i}=z_{i}
\end{array}\right.
$$

Where, $\theta$ is the angle between the pick coordinate vector and the positive direction of the horizontal axis in the plane ${ }_{4} Y_{4} Z_{4}$ and $\theta \in[0,2 \pi]$, ri is i-th pick relative to the $Z_{4}$ axis radius.

Simultaneous equations (1) (3) (4) (5) get the curve equation of pick's cutting track in the bodywork's coordinate system.

$$
\left\{\begin{array}{l}
{ }^{0} x_{i}=a_{2} c \theta_{1}+b_{3} c \theta_{1} c \theta_{2}+r_{i} c \theta s \theta_{1}-z_{i} c \theta_{2} s \theta_{2}-\left(a_{3}+a_{4}+d\right) c \theta_{1} s \theta_{2}+r_{i} c \theta_{1} c \theta_{2} s \theta \\
0 y_{i}=a_{2} s \theta_{1}+b_{3} c \theta_{2} s \theta_{1}-r_{i} c \theta c \theta_{1}-z_{i} s \theta_{1} s \theta_{2}-\left(a_{3}+a_{4}+d\right) s \theta_{1} s \theta_{2}+r_{i} s \theta_{1} c \theta_{2} s \theta \\
{ }^{0} z_{i}=-b_{3} s \theta_{2}-z_{i} c \theta_{2}-\left(a_{3}+a_{4}+d\right) c \theta_{2}-r_{i} s \theta_{2} s \theta
\end{array}\right.
$$

Projection equation in the vehicle coordinate plane is:

$\left\{\begin{array}{l}{ }^{0} y_{i}=a_{2} s \theta_{1}+b_{3} c \theta_{2} s \theta_{1}-r_{i} c \theta^{\prime} c \theta_{1}-z_{i} s \theta_{1} s \theta_{2}-\left(a_{3}+a_{4}+d\right) s \theta_{1} s \theta_{2}+r_{i} s \theta_{1} c \theta_{2} s \theta^{\prime} \\ 0\end{array}\right.$

Where, $\theta^{\prime}$ is the angle between the pick coordinate vector and the positive direction of the horizontal axis in the plane $O_{0} Y_{0} Z_{0}$ and $\theta^{\prime} \in[0,2 \pi]$.

By equations (7), we can see that in addition to the cutting head center at the origin of coordinates, with the changes of $\theta_{1}$ and $\theta_{2}$, the projection equation of pick's cutting track in the vehicle coordinate plane are ellipse with different shape. 


\section{The Determination of the Inflection Point Position in the Process Path}

C. Rectangular section

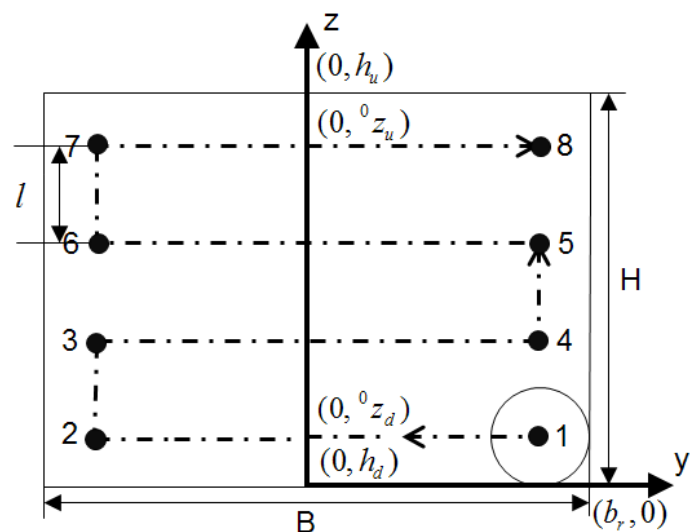

Figure 4. The determination of inflection point in the rectangular section

The cutting range of the cantilever left swing, downward swing and upward swing was analyzed in the literature [9]. When the cutting head cutting the upper boundary, we put $\theta=\frac{p i}{2}$ into (6), and get the

${ }^{0} z_{i}=-\left(r_{i}+b_{3}\right) s \theta_{2}-\left[z_{i}+a_{3}+a_{4}+d\right] c \theta_{2}$

When the cutting head cutting the lower boundary, we put $\theta=\frac{3 p i}{2}$ into (6), and get the

${ }^{0} z_{i}=\left(r_{i}-b_{3}\right) s \theta_{2}-\left[z_{i}+a_{3}+a_{4}+d\right] c \theta_{2}$.

Putting $h_{u}$ and $h_{d}$ which is the ordinate of the rectangular section's upper and lower boundary into (8) and (9) find out the corresponding cantilever swing angle $\theta_{2}$. Further, the ordinate ${ }^{0} z_{u}$ and ${ }^{0} z_{d}$ of the cutting head center can be calculated by (2) (see fig. 4).

Setting the given reference step length is $l^{\prime}$, the actual step length can be calculated by (10).

$$
l=\frac{{ }^{0} z_{u}-{ }^{0} z_{d}}{\operatorname{ceil}\left(\frac{{ }^{0} z_{u}-{ }^{0} z_{d}}{l}\right)}
$$

The number of the inflection point in the whole process path is:

$$
j=2\left[\operatorname{ceil}\left(\frac{{ }^{0} z_{u}-{ }^{0} z_{d}}{l^{\prime}}\right)+1\right]
$$

The ordinate of all the inflection point is:

$z_{2 n+1}=z_{2 n+2}={ }^{0} z_{u}+n l$

Where $n=0,1,2, \ldots, \operatorname{ceil}\left(\frac{{ }^{0} z_{u}-{ }^{0} z_{d}}{l^{\prime}}\right)$.

We discuss only the situation when the cutting head cuts the section's right boundary. Put $\theta=0$ into (6) and get the

$$
{ }^{0} y_{i}=r_{i} c \theta_{1}+\left[b_{3} c \theta_{2}-\left(z_{i}+a_{3}+a_{4}+d\right) s \theta_{2}+a_{2}\right] s \theta_{1} .
$$

Putting the corresponding cantilever swing angle $\theta_{2}$ of the inflection point near the right boundary $(1,4,5,8)$ and the abscissa ${ }{ }_{r}$ of the rectangular section's right boundary into (13) find out the cantilever swing angle ${ }^{\theta_{1}}$.Further, the abscissa ${ }^{y_{j}}$ of the cutting head center can be calculated by (2).

In this way, it can calculate all the position of the inflection point on the process path of the rectangular section automatic forming. 


\section{Trapezoidal section}

When the cutting head cut bevel edge of trapezoidal, the angle $\theta \neq 0, \frac{p i}{2}, \frac{3 p i}{2}$ between the pick coordinate vector and the positive direction of the horizontal axis, so inflection point cannot be determined by the above method. This paper uses "approximate iterative method" to determine the position of the inflection point for the trapezoidal section. The solving method for $\theta_{2}$ corresponding to the each inflection point position is the same as rectangular section. Taking the first inflection point as an example, the method is as follows.

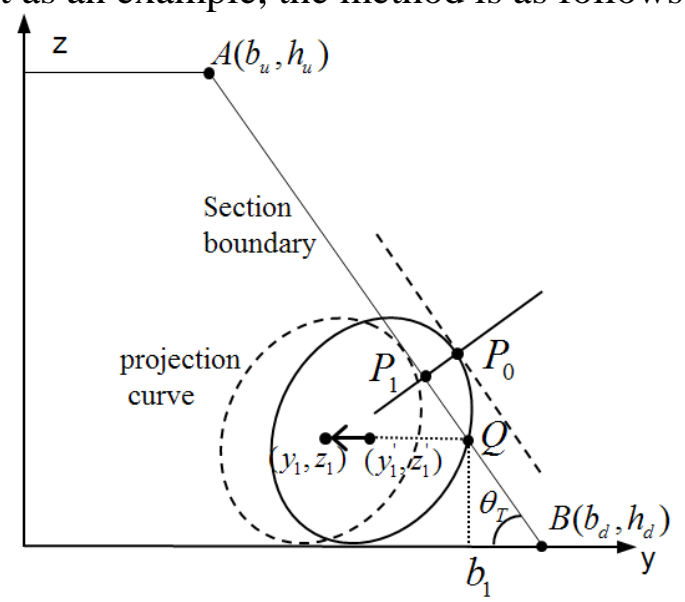

Figure 5. The determination of inflection point in the trapezoidal section

1) Preset the iteration variable of the initial value

First, determine the corresponding boundary point of the first point Q (see fig.5).

Equation of the trapezoidal bevel is:

$\left(h_{d}-h_{u}\right) y-\left(b_{d}-b_{u}\right) z+\left(h_{u}-h_{d}\right) b_{d}=0$

Where, $b_{u}, b_{d}, h_{d}, h_{u}$ as shown in fig. 5

Putting the ordinate of the first inflection point into (14) find out the abscissa ${ }^{b_{1}}$ of the boundary point Q. And putting ${ }^{b_{1}}$ and $\theta_{2}$ of the first inflection point into (13) find out the $\theta_{1}$ of the first inflection point, and as the initial value assigned to the ${ }^{\theta_{1}(0)}$.

2) The establishment of the iterative formula

Derivation of the (7), derivative of the cutting trajectory of the pick in the plane is:

$\frac{d^{0} z_{i}}{d^{0} y_{i}}=-\frac{r_{i} c \theta^{\prime} s \theta_{2}}{r_{i} c \theta_{1} s \theta^{\prime}+r_{i} c \theta_{2} c \theta^{\prime} s \theta_{1}}$

Corner of the trapezoid section:

$\theta_{T}=a \tan \left(\frac{b_{u}-b_{d}}{h_{u}-h_{d}}\right)$

Let $-\frac{r_{i} c \theta^{\prime} s \theta_{2}}{r_{i} c \theta_{1} s \theta^{\prime}+r_{i} c \theta_{2} c \theta^{\prime} s \theta_{1}}=\tan \theta_{T}$, Put $\theta_{1}(0)$ and $\theta_{2}$ into equation to find $\theta^{\prime} \quad\left(\operatorname{take} \theta^{\prime}<p i\right)$.

Putting $\theta_{1}, \theta_{2}$ and $\theta^{\prime}$ into (7) to get point $P_{0}\left[{ }^{0} y_{i q},{ }^{0} z_{i q}\right]$ of which curvature in the projection curve is equal to the slope of the trapezoidal bevel. This is the maximum cutting point of pick.

The normal equation of the trapezoidal bevel is:

$\left(b_{d}-b_{u}\right) y-\left(h_{u}-h_{d}\right) z+m=0$

Put point $\left[{ }^{0} y_{i q},{ }^{0} z_{i q}\right]$ into (17) to get parameter m.

Simultaneous equations (13) (14) solve the coordinates $P_{1}\left[{ }^{0} y_{i j},{ }^{0} z_{i j}\right]$ which is the intersection of two straight lines. The modified inflection point coordinate can be got by the cutting head center coordinates move with the vector $\left[{ }^{0} y_{i j}{ }^{0} y_{i q}, 0\right]$. New ${ }^{\theta_{1}(1)}$ can be calculated by (2).

3) To control the iterative process

The objective function is defined as: 


$$
F=\sqrt{\left({ }^{0} y_{i q}-{ }^{0} y_{i j}\right)^{2}+\left({ }^{0} z_{i q}-{ }^{0} z_{i j}\right)^{2}} \text {. }
$$

The change of the pick's projection curve will be further caused in the plane $O_{0}-Y_{0} Z_{0}$ when the center position of the cutting head changes. When the trapezoidal section base angle is small, only one correction for inflection point on the process path cannot achieve precise cutting. Repeat the above process, iteration process will be end when the objective function $\mathrm{F}$ is less than the preset error. The corresponding cantilever swing angle $\theta_{1}$ of the entire inflection point can be obtained by this method.

\section{The Simulation and Verification}

Taking EBJ-132 boom-type roadheader as an example, MATLAB can be used for simulation and verification of section automatic forming. The basic parameters of EBJ-132 roadheader: $a_{1}=1145 \mathrm{~mm}, b_{1}=720 \mathrm{~mm}, \quad a_{2}=720 \mathrm{~mm}, \quad b_{2}=80 \mathrm{~mm}, \quad a_{3}=2050 \mathrm{~mm}, \quad b_{3}=100 \mathrm{~mm}$, $\theta_{1}=-39^{\circ} \sim+39^{\circ}, \theta_{2}=-31^{\circ} \sim+42^{\circ}, d=0 \sim 800 \mathrm{~mm}$. Set pick i which forms the section boundary in the cutting head relative to the $\mathrm{Z}$ axis's radius is $300 \mathrm{~mm}$, zi $=-200 \mathrm{~mm}$. Set step length is $500 \mathrm{~mm}$ and the cutting precision is $1 \mathrm{~mm}$.

Set trapezoidal roadway section's under width $\mathrm{x}$ upper width $\mathrm{x}$ height is $3000 \mathrm{~mm} \mathrm{x} 1800 \mathrm{~mm} \mathrm{x}$ $1800 \mathrm{~mm}$. The simulation map of the trapezoidal section automatic cutting process path as shown in fig.6

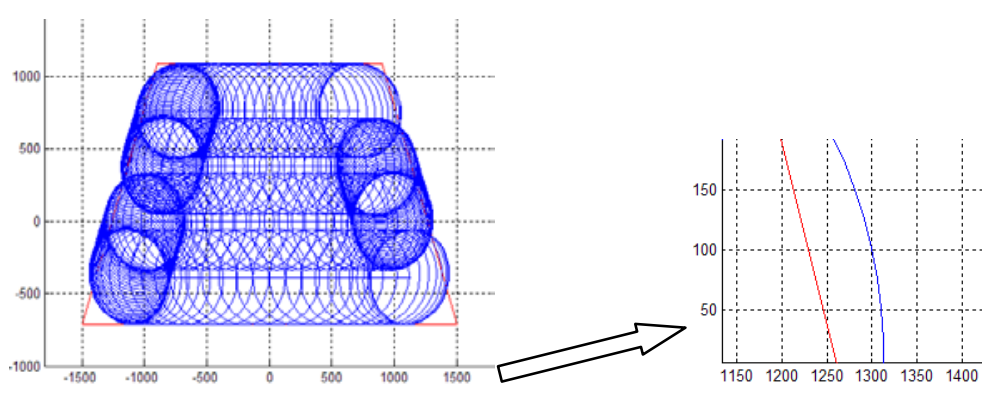

(a) Adopt the literature [9] method

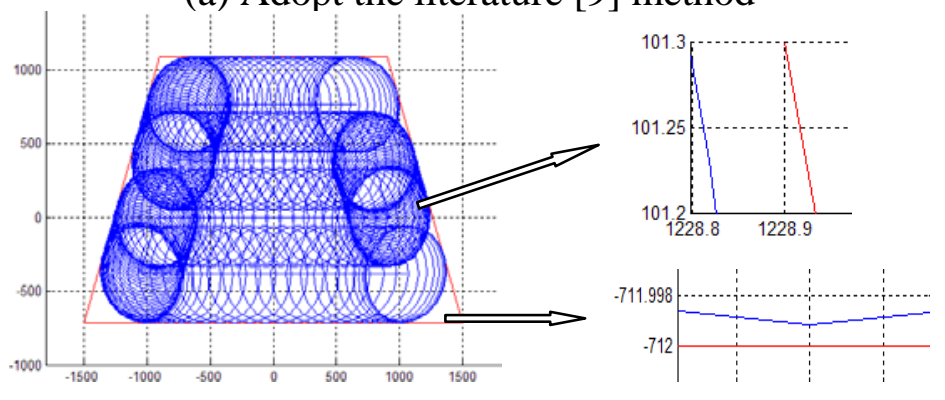

(b) Approximate iterative method

Figure 6. The simulation diagram of process path

Fig.6 shows that using the rectangular section method to determine process path will produce obvious extra cutting (usually reach $100 \mathrm{~mm}$ ). However, thought this paper method to calculate the inflexion position and determine the process path can achieve precise forming of section (the cutting error can be controlled within $1 \mathrm{~mm}$ ).

\section{Conclusions}

1) The cutting precision model was set up for the first time to trapezoidal section;

2) Taking the cutting precision as the objective function "approximate iterative method" was used to find inflection point to trapezoidal section in automatic forming process path;

3) The algorithm is implemented by using MATLAB, and proved to be correct through the simulation on trapezoidal cross section; 
4) This method is applicable not only to trapezoidal section, but also can be extended to nonrectangular section, and has a certain universal.

\section{References}

[1] Tong Minming, Kang Dongliang, Liu Peng,"Research on Automatic Section Cutting Control of Roadheader,” 2010 International Conference on Measuring Technology and Mechatronics Automation.2010,pp.22-25

[2] Huang Riheng, “Boom-type Roadheader,” Beijing, China University of Mining and Technology press, 1996

[3] Cai Zixing, "Fundamentals of Robotics,” Beijing, Machinery Industry Press, 2009

[4] Tian Jie, “Automatics Section Cutting and Forming Control of Longitudinal-axial-roadheaders," Journal of China Coal Society. 2009,34(1),pp.111-115

[5] Zhang Shiyong, Ning Zhongliang, "Application of MATLAB in boom-type roadheader auto profiling control research,” Coal Mine Machinery.2005,11,pp.87-89

[6] Tian Jie, "Reasearch on boom-type roadheader auto cutting and profiling control system," Beijing, China University of Mining and Technology,2009

[7] Xiong Shuanghui,"Study on boom-type roadheader laneway section shaping control system," Xian, Xi'an University of Science And Technology,2009

[8] Chen Jiasheng, Zhang Zhikang, Deng Haishun,"MATLAB simulation of intelligent control cutting of boom-type roadheader,” Mining Technology,2007,7(3),pp.38-42

[9] Li Junli, "Reserch on Automatic Cross Section Profiling Theory And Control Strategy for Boom-Type Roadheader,” Taiyuan, Taiyuan University of Technology,2009 